\title{
Prevalence of Cryptosporidium species among immunocompetent and immunocompromised Egyptian children: comparative study \\ Original
} Article

\author{
Aliaa M Elsawey ${ }^{1}$, Suzan H Elgendy ${ }^{1}$, Salama A Abdel-Magied ${ }^{1}$, Yousef Mosaad ${ }^{2}$, \\ Nairmen Nabih ${ }^{1}$
}

Departments of Medical Parasitology ${ }^{1}$ and Clinical Pathology², Faculty of Medicine, Mansoura University

\begin{abstract}
Background: Cryptosporidium is a protozoan parasite that causes gastrointestinal infection. Each cryptosporidial species usually inhabits a particular host; however cross-infectivity could occur.

Objectives: The aim of the study was to identify prevalent Cryptosporidium species among a sample of immunocompetent and immunocompromised children.

Subjects and Methods: This study included 150 children divided as 50 control group (apparently healthy with no complaints) and 100 patients (immunocompetent and immunocompromised) with gastrointestinal manifestations. Stool samples were collected and examined microscopically using modified Zeihl-Neelsen stain (mZN), ELISA for coproantigen and nested PCR (nPCR). Restriction fragment length polymorphism (RFLP) technique was done for species identification of PCR products.

Results: Among the 100 patients, cryptosporidiosis prevalence was $34 \%, 46 \%$ and $59 \%$ as detected by mZN, ELISA and nPCR, respectively. Infection was more prevalent in immunocompromised group (84\%) than immunocompetent group $(34 \%)$ as detected by nPCR $(P<0.001)$. Cryptosporidiosis was found to be significantly associated with nausea $(P=0.002)$ and with diarrhea $(P=0.04)$. Detected species among studied children were C. hominis (52.5\%), C. parvum (33.9\%), C. meleagridis (8.5\%) and C. felis (5.1\%). C. hominis was the prevalent species in both immunocompetent and immunocompromised groups.

Conclusion: $C$. hominis was proven to be the more prevalent species among cryptosporidiosis positive children in this study. More attention should be paid to this emerging parasitic infection especially in immunocompromised children.
\end{abstract}

Keywords: Cryptosporidium spp., Egypt, nested PCR, prevalence, RFLP.

Received: 11 April, 2020, Accepted: 18 June, 2020.

Corresponding Author: Aliaa Elsawey, Tel.: +20 1008874025, E-mail: aliaaelsawey@gmail.com

Print ISSN: 1687-7942, Online ISSN: 2090-2646, Vol. 13, No. 2, August, 2020.

\section{INTRODUCTION}

Cryptosporidium is a protozoan inhabitant of the brush borders of the gastrointestinal epithelial cells. It was thought to be a zoonotic pathogen; however, anthroponotic transmission was also established ${ }^{[1]}$. Infection results from consumption of contaminated water or food including raw milk ${ }^{[2]}$. Generally, cryptosporidiosis is a short-term sickness in the form of diarrhea and weight loss in immunocompetent children and adults. However, in immunocompromised individuals, infection could be prolonged and lifethreatening ${ }^{[3]}$. A variable prevalence range of $6 \%$ to $49 \%$ was recorded in different reports on the infection in Egyptian children ${ }^{[4-8]}$.

Molecular techniques for differentiation of species by detection of the small subunit ribosomal ribonucleic acid (SSU rRNA) of Cryptosporidium oocyst wall protein genes ${ }^{[9]}$ were reviewed. C. hominis and $C$. parvum were recorded as the major species infecting human population. Other species, like $C$. felis, C. meleagridis, C. muris, C. canis and C. suis were reported less frequently. Infection with $C$. hominis was linked to different manifestations as diarrhea, nausea, vomiting, general malaise, while infection with C. parvum was linked mainly to cases with diarrhea ${ }^{[10,11]}$. El-Hamshary et al. ${ }^{[4]}$, in an early study on immunocompromised patients of different ages from attendants of Suez Canal University Hospitals in Egypt, used multiplex allele specific polymerase chain reaction and recorded $68.4 \%$ infection with $C$. parvum and $26.3 \%$ with $C$. hominis. Moreover, $C$. parvum was found to be more prevalent (61.5\%) in rural localities while C. hominis was more prevalent in urban areas (60\%). In another attempt to genotypically characterize Cryptosporidium spp. in a sample of isolates from calves and children suffering from diarrhea, $88.9 \%$ from the children's positive samples were identified as $C$. hominis ( $C$. parvum genotype 1) and genotype 2 C. parvum was apparently more prevalent in the sample of calves ${ }^{[12]}$.

Our study was conducted to identify the prevalent Cryptosporidium species among immunocompetent and immunocompromised children attending Mansoura University Children's Hospital in Egypt. 


\section{SUBJECTS AND METHODS}

Subjects: The present case-control study was conducted in laboratories of Mansoura Faculty of Medicine, Egypt during the period from April 2016 to September 2017. One hundred and fifty children aged from 2 to 17 years were included. One hundred children were patients attending different departments of Mansoura University Children's Hospital. They were divided into two groups (each of 50) to include (1) immunocompetent children complaining of gastrointestinal manifestations, (2) immunocompromised children due to chemotherapy for hematopoietic and lymphatic tumors and complaining of gastrointestinal manifestations. In addition, 50 children included as control group were selected from healthy visitors or were accompanying patients attending the hospital for other complaints. All children were subjected to a questionnaire concerning their medical history, and clinical examination.

Sample collection and microscopic examination: Stool samples were collected (from all participated children) in clean, dry, labeled plastic containers and divided into three parts; first part for concentration by formalin-ether technique, followed by $\mathrm{mZN}^{[13]}$, and microscopic examination for oocysts. The second and third parts, without any additives, were stored at $-20^{\circ} \mathrm{C}$ for coproantigen detection and molecular processing, respectively.

ELISA technique for coproantigen: ELISA technique was performed for detection of Cryptosporidium coproantigen (Epitope Diagnostic's, Inc., San Diego, USA) according to the manufacturer's instructions ${ }^{[14]}$.

Nested PCR: DNA was extracted from frozen fecal specimens using QIAamp DNA stool mini kit (Qiagen, Biocompare, USA) according to the manufacturer's protocols $^{[15,16]}$. nPCR technique was carried out to amplify SSU rRNA gene of Cryptosporidium ${ }^{[17,18]}$. This technique amplifies a $~ 830$-bp fragment of the SSU rRNA gene by nPCR and differentiates Cryptosporidium species by banding patterns in restriction analysis of the secondary PCR products with the enzymes SspI and VspI. The primary PCR and secondary PCR reactions were followed by running $20 \mu \mathrm{L}$ of the PCR product for electrophoresis on a $1.5 \%$ agarose ge ${ }^{[18]}$. The primers (Bio Basic Canada Inc., Markham, ON, Canada) are listed in table (1).

Table 1. Primers used in the nPCR reactions

\begin{tabular}{lll}
\hline \hline Primary & Forward (F1) & 5'-TTCTAGAGCTAATACATGCG-3' \\
primers & Reverse (R1 & 5'-CCCATTTCCTTCGAAACAGGA-3' \\
\hline $\begin{array}{l}\text { Secondary } \\
\text { primers }\end{array}$ & Forward (F1) & 5'-GGAAGGGTTGTATTTATTAGATAAAG-3' \\
\hline \hline
\end{tabular}

RFLP technique: Restriction enzymes used were SspI (Enzynomics co. Ltd, Daejeon, Republic of Korea) and VspI (Thermo Fisher Scientific, Massachusetts,
USA). Electrophoresis was run on agarose gel with restriction digestion reaction whole volume of $40 \mu \mathrm{L}$, and Cryptosporidium species were identified based on RFLP banding patterns. Species diagnosis was made by digesting the secondary PCR product (826 to $864 \mathrm{bp}$ ) with SspI, and VspI according to Xiao et al. ${ }^{[18]}$.

Statistical analysis: Analysis of data was done using IBM SPSS software package version 20.0 (IBM, Chicago, IL, USA). Categorical variables were analyzed by Kruskal-Wallis $\mathrm{H}$ and Mann-Whitney $\mathrm{U}$ tests. Chi Square test was used when appropriate. $P$ value $<0.05$ was considered statistically significant at confidence interval 95\%. Logistic regression analysis was performed for the association of cryptosporidiosis as a risk factor for the occurrence of gastrointestinal symptoms.

Ethical approval: Informed consent was obtained from the parents of all participated children. All performed procedures were in accordance with Helsinki declaration. Ethics Review Committee and the Institutional Review Board of the Faculty of Medicine, Mansoura University, Egypt, approved this study with code number $\mathrm{R} / 15.06 .26$. Infected children were referred to pediatric staff for appropriate treatment with antiprotozoal agent.

\section{RESULTS}

Among studied children with gastrointestinal manifestations $\quad(n=100), \quad 34 \% \quad$ harbored Cryptosporidium oocysts, identified by mZN stain (Table 2). Using ELISA, 46\% were positive while by nPCR, 59\% were positive (Fig. 1). Among control children $(n=50)$, the recorded prevalence was $4 \%, 6 \%$ and $10 \%$ as detected by mZN stain, ELISA and nPCR respectively. Statistically relevant cryptosporidiosis $(P<0.001)$ was more prevalent in immunocompromised group (50\%, 66\% and 84\% respectively) than immunocompetent group $(18 \%$, $26 \%$ and $34 \%$, respectively) (Table 2).

Infection was significantly higher in children aged less than 5 years old $(P<0.001)$. No difference was recorded regarding gender or between urban and rural areas. Gastrointestinal manifestations among studied cases $(n=100)$ showed that nausea and diarrhea were significantly high in Cryptosporidium infected group $(P<0.05)$. Cryptosporidium spp. was detected in 31/49 diarrheic children (63.3\%). By Likelihood ratio tests among different symptoms, nausea and diarrhea were significantly related to cryptosporidiosis, $P=0.002$ and 0.04 respectively (Table 3). Cryptosporidiosis was a potential risk factor for nausea in children, with an odds ratio (OR) $=3.9(95 \% \mathrm{CI}=1.6$ to 9.6 and $P<0.01)$; and also for diarrhea in children, OR $=2.3(95 \% \mathrm{CI}=1.02$ to 5.22 and $P<0.05$ ). 
PARASITOLOGISTS UNITED JOURNAL

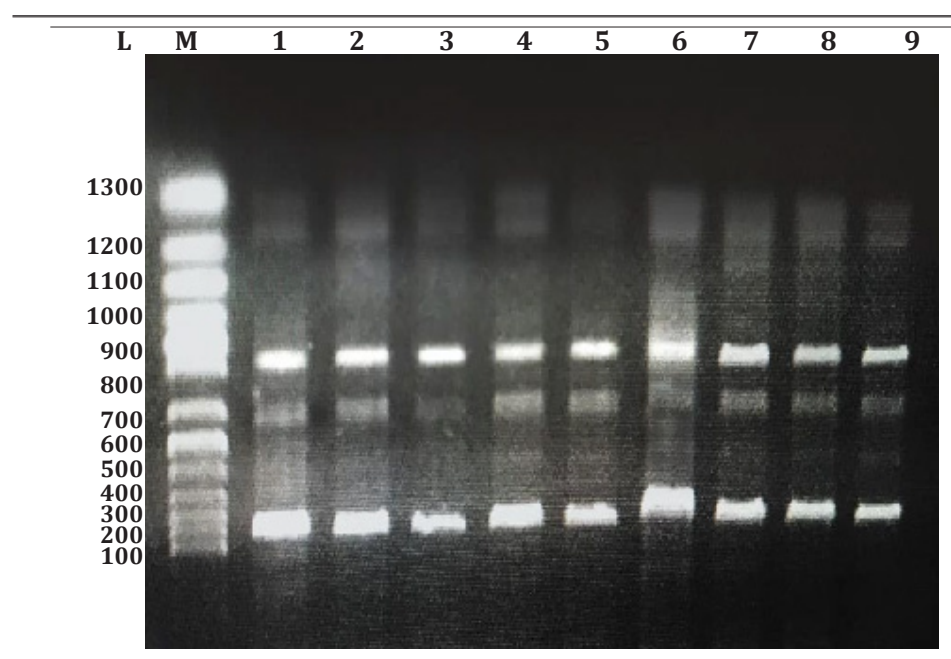

Fig. 1 Diagnosis of cryptosporidiosis by nPCR procedure based on detection of the 830-bp SSU rRNA gene. M, Molecular markers of 100-bp ladders.

Lane 1-9, Amplified 830-bp of 18s rRNA gene for Cryptosporidium.

Table 2. Prevalence of Cryptosporidium infection among controls and patients using mZN, ELISA and nPCR techniques.

\begin{tabular}{lccc}
\hline \multirow{2}{*}{ Group } & \multicolumn{3}{c}{ Method used } \\
\cline { 2 - 4 } & mZN Microscopy & ELIZA & nPCR \\
\cline { 2 - 4 } & No. (\%) & No. (\%) & No. (\%) \\
\hline Control group (n=50) & $2(4)$ & $3(6)$ & $5(10)$ \\
Patients group (n=100) & $34(34)^{*}$ & $46(46)^{*}$ & $59(59)^{*}$ \\
\hline Immunocompetent patients (n=50) & $9(18)$ & $13(26)$ & $17(34)$ \\
Immunocompromised patients (n=50) & $25(50)^{* *}$ & $33(66)^{*}$ & $42(84)^{*}$ \\
\hline \hline
\end{tabular}

${ }^{*} P<0.001,{ }^{* *} P=0.001$, mZN: modified Zeihl Neelsen, nPCR: nested PCR

Table 3. Likelihood ratio results of symptoms among patient groups in relation to cryptosporidiosis ( $\mathrm{n}=100$ ).

\begin{tabular}{lcc}
\hline \multirow{2}{*}{ Symptoms } & \multicolumn{2}{c}{ Likelihood ratio tests } \\
\cline { 2 - 3 } & Chi-Square & Significance $(\boldsymbol{P}$ value $)$ \\
\hline Nausea & 9.808 & $0.002^{*}$ \\
Vomiting & 2.500 & 0.114 \\
Diarrhea & 4.136 & $0.042^{*}$ \\
Abdominal pain & 0.268 & 0.605 \\
Weight loss & 2.925 & 0.087 \\
\hline \hline
\end{tabular}

$\overline{* P<0.05}$

Among samples proved positive by nPCR, RFLP identified C. hominis (52.5\%), C. parvum (33.9\%), C. meleagridis (8.5\%) and C. felis (5.1\%). Comparing Cryptosporidium species distribution between infected cases in immunocompetent and immunocompromised groups showed no significant differences between the two groups. $C$. hominis had the highest frequency in both groups (Table 4). Gastrointestinal symptoms among infected group $(n=59)$ showed regression analysis of the association between $C$. hominis and gastrointestinal manifestations (Table 5), except for a significant association with nausea symptom, $(\mathrm{OR})=20.125(95 \% \mathrm{CI}=2.133$ to 189.879 and $P=$ 0.009).

Table 4. Cryptosporidium species distribution among Cryptosporidium-positive patients (immunocompetent and immunocompromised).

\begin{tabular}{|c|c|c|}
\hline \multirow[b]{2}{*}{ Symptoms } & \multicolumn{2}{|c|}{ Patient group* } \\
\hline & $\begin{array}{c}\text { Immunocompetent }(\mathrm{n}=17) \\
\text { No. }(\%)\end{array}$ & $\begin{array}{c}\text { Immunocompromised }(\mathrm{n}=42) \\
\text { No. }(\%)\end{array}$ \\
\hline C. hominis & $10(58.8)$ & $21(50.0)$ \\
\hline C. parvum & $5(29.4)$ & 15 (35.7) \\
\hline C. meleagridis & $1(5.9)$ & $4(9.5)$ \\
\hline C. felis & $1(5.9)$ & $2(4.8)$ \\
\hline
\end{tabular}

$* P>0.05$ between the two groups for detected species. 
Cryptosporidium species among children

\begin{tabular}{|c|c|c|c|c|}
\hline \multirow{2}{*}{ Clinical findings } & \multicolumn{4}{|c|}{ Detected Cryptosporidium species } \\
\hline & C. hominis $(\mathrm{n}=31)$ & C. parvum $(\mathrm{n}=20)$ & C. meleagridis $(\mathrm{n}=5)$ & C. felis $(\mathrm{n}=3)$ \\
\hline \multicolumn{5}{|l|}{ Symptoms\# } \\
\hline Nausea & $28(90.3 \%)^{*}$ & $4(20 \%)$ & $1(20 \%)$ & $1(33.3 \%)$ \\
\hline Vomiting & $21(67.7 \%)$ & $7(35 \%)$ & $1(20 \%)$ & $1(33.3 \%)$ \\
\hline Diarrhea & $23(74.2 \%)$ & $5(25 \%)$ & $2(40 \%)$ & $1(33.3 \%)$ \\
\hline Abdominal pain & $15(48.4 \%)$ & $11(55 \%)$ & $3(60 \%)$ & $0(0 \%)$ \\
\hline Weight loss & $13(41.9 \%)$ & $8(40 \%)$ & $3(60 \%)$ & $0(0 \%)$ \\
\hline \multicolumn{5}{|l|}{ Signs } \\
\hline Tender abdomen & $15(48.4 \%)$ & $9(45 \%)$ & $2(40 \%)$ & $0(0 \%)$ \\
\hline Dehydration & $8(25.8 \%)$ & $8(40 \%)$ & $3(60 \%)$ & $0(0 \%)$ \\
\hline Hepatomegaly & $6(19.4 \%)$ & $5(25 \%)$ & $2(40 \%)$ & $0(0 \%)$ \\
\hline Others & $11(35.5 \%)$ & $6(30 \%)$ & $1(20 \%)$ & $1(33.3 \%)$ \\
\hline
\end{tabular}

\section{DISCUSSION}

Cryptosporidium has been considered as one of the main pathogens responsible for severe diarrhea and deaths in infants ${ }^{[19,20]}$. Investigating the cause of moderate-to-severe diarrhea in children under 2 years old, four pathogens were responsible for the majority of cases; rotavirus, Cryptosporidium, Shigella spp., and heat-stable toxin producing enterotoxigenic Escherichia coli ${ }^{[21]}$. Cryptosporidium is transmitted via ingestion of oocysts-contaminated food and water or directly through contact with infected person or animal. Following oocysts ingestion, sporozoites are released and infect the host's intestinal epithelial cells, leading to various gastrointestinal manifestations ${ }^{[10]}$.

Cryptosporidiosis generally causes a short-term sickness in immunocompetent persons; however, in immunocompromised persons, the infection could be prolonged due to excystation of thin-shelled oocysts and occurrence of internal autoinfection ${ }^{[22]}$. The overall infection prevalence in our study was 34\%, 46\% and $59 \%$ as detected by mZN, ELISA and nPCR respectively. According to Omoruyi et al. ${ }^{[23]}$, detection percentages of cryptosporidiosis by mZN, ELISA and nPCR diagnostic techniques were $37.1 \%, 74.3 \%$ and $65.7 \%$ respectively in HIV-positive patients, while in HIV-negative persons, the detection percentage was $27.2 \%, 76.8 \%$ and $71.2 \%$ respectively.

In the present study, prevalence of Cryptosporidium infection in studied cases was $59 \%$ as detected by nPCR technique, approaching the report by Helmy et $a l^{\left[{ }^{[6]}\right.}$ of 49.1\% prevalence in Egyptian children but higher than that by El-Badry et al. ${ }^{[8]}$ who recorded a prevalence of 19.5\%. Both reports involved Egyptian children. In our study prevalence of cryptosporidiosis was significantly higher in immunocompromised children (84\%) than immunocompetent ones (34\%) and apparently healthy controls (10\%), signifying previous suggestions recommending the important role of immunity in controlling this infection ${ }^{[24]}$. Higher prevalences among immunocompromised patients due to different causes was also recorded in other studies ${ }^{[25,26]}$.
Cryptosporidiosis has great impact on public health due to occupational risks represented by asymptomatic food workers who are considered responsible for asymptomatic carriage ${ }^{[27,28]}$. Major food-borne cryptosporidial outbreaks among general populations without restriction to immunocompromised patients, were reported in USA and Sweden ${ }^{[29,30]}$.

We detected a significantly higher prevalence in children aged less than 5 years old conforming with other studies; attributing this to an immature intestinal tract mucosal immunity, unhygienic behavior with high exposure and improper hand washing in this age $\mathrm{e}^{[5,31,32]}$. In an important observation, Hsu et al. remarked that Cryptosporidium oocysts expressed high resistance to chlorine disinfection, indicating the importance of boiling potable water in combatting this parasite ${ }^{[33]}$.

Cryptosporidium infection was recorded as relatively high in the age group of 13-36 months ${ }^{[34]}$. In contrast, the prevalence rates detected in USA, were lowest in younger children and progressively increased in older ages ${ }^{[35]}$. This was attributed to the greater risk of exposure in elderly persons in developed countries. No significant link to residence was detected in our study suggesting that different methods of transmission as direct contact and water borne infection may be responsible in both rural and urban areas ${ }^{[36]}$.

The principal symptom of cryptosporidiosis is diarrhea, in addition to other gastrointestinal manifestations. In children, especially in developing countries, the course of diarrhea could last for two weeks or more ${ }^{[32]}$. Among the immunocompetent and immunocompromised children enrolled in our study Cryptosporidium oocysts were detected in 63.3\% complaining of diarrhoea, confirming the susceptibility of children to this infection. This is substantiated by Abdel Gawad et al. ${ }^{[36]}$ who reported a lower rate of infection in an older age group of $37 \pm 16.8$ years complaining of diarrhea. We recorded a significantly higher occurrence of nausea in Cryptosporidium- 
infected group (57.6\%), consistent with Adler et $a l^{[22]}$ who reported a significant association between Cryptosporidium infection and nausea.

Cryptosporidium hominis and C. parvum were recorded as the major species infecting human population. Other species, like $C$. felis, $C$. meleagridis, C. muris, C. canis and C. suis were reported less frequently ${ }^{[10,11]}$. We verify the former reports by identifying four of the Cryptosporidium species; C. hominis, C. parvum, C. meleagridis and C. felis. The prevalent species among both infected immunocompetent and immunocompromised groups was $C$. hominis, suggesting anthroponotic transmission of human cryptosporidiosis in Mansoura City and nearby cities and rural areas. High prevalence of $C$. hominis was also reported in previous studies from Egypt $^{[5,8,37]}$.

According to Sadek ${ }^{[12]}$, about $88.9 \%$ of stool samples proved positive in studied children from the Gastroenteritis Unit, Abo El Reesh Pediatric Hospital, Cairo University, were identified as genotype 1 (C. hominis). Also from Egypt but from a different locality, El-Hamshary et al. ${ }^{[4]}$ showed that most of the detected Cryptosporidium species in children from Suez Canal University Hospital, were C. parvum (68.4\%); while C. hominis was detected in only $26.3 \%$ of positive cases. Similar prevalence was recorded from immunocompromised inpatients of the oncology, nephrology and pediatrics departments, Suez Canal University hospitals by Soliman and Othman ${ }^{[38]}$. Using multiplex PCR the authors identified C. parvum in 64.7\% and $C$. hominis in $29.4 \%$ of positive samples. From other countries (Kuwait and Iran) C. parvum was the more prevalent species ${ }^{[39,40]}$. This is understandable because C. parvum can infect a range of different species, while C. hominis appears to be a particular human parasite with more virulent characteristics than the general C. parvum $^{[41]}$. Other Cryptosporidium species with different host ranges also could infect human ${ }^{[42]}$.

Logistic regression analysis of our results revealed that, among the detected Cryptosporidium species, C. hominis was significantly associated with nausea symptom and may therefore be considered as a risk factor for development of nausea in children. Gastric involvement is thought to occur subsequent to small intestine colonization. It was suggested that conditions associated with hypochlorhydria as in HIV/AIDS, predisposes to gastric cryptosporidial colonization ${ }^{[43]}$. However, gastric cryptosporidiosis was also reported in immunocompetent patients ${ }^{[4,45]}$. A small infectious dose of 10-30 oocysts was implicated in the possibility of infection among immunocompetent individuals ${ }^{[46]}$. Cryptosporidiosis was suggested as a potential cause of unexplained symptoms in infected persons ${ }^{[47]}$, as gastrointestinal symptoms can persist for long periods after the initial infection ${ }^{[48,49]}$.
Our study concludes the high prevalence of $C$. hominis among a sample of children attending Mansoura University Children's Hospital, and associates it mainly with the symptom of nausea. The inclusion of special stains as mZN in routine stool examination is needed for preliminary detection of this emerging parasitic infection.

Author contributions: Abdel Magied SA initiated and designed the study; Elgendy $\mathrm{SH}$ collected samples and shared with Elsawey A and Nabih $\mathrm{N}$ in performance of laboratory work and writing; Mosaad Y supervised laboratory work.

Conflict of interests: The authors declare that there is no conflict of interests.

\section{REFERENCES}

1. Checkley W, White AC Jr, Jaganath D, Arrowood MJ, Chalmers RM, Chen XM, et al. A review of the global burden, novel diagnostics, therapeutics, and vaccine targets for Cryptosporidium. Lancet Infect Dis 2015; 15(1):85-94.

2. Åberg R, Sjöman M, Hemminki K, Pirnes A, Räsänen S, Kalanti A, et al. Cryptosporidium parvum caused a large outbreak linked to Frisée salad in Finland, 2012. Zoonoses Public Health 2015; 62(8): 618-624.

3. Chalmers RM, Davies AP. Minireview: clinical cryptosporidiosis. Exp Parasitol 2010; 124(1):138-146.

4. El-Hamshary EM, El-Sayed HF, Hussein EM, Rayan HZ, Soliman RH. Comparison of polymerase chain reaction, immunochromatographic assay and staining techniques in diagnosis of cryptosporidiosis. PUJ 2008; 1(2):77-86.

5. Abd El Kader NM, Blanco MA, Ali-Tammam M, Abd El Ghaffar AERB, Osman A, El Sheikh N, et al. Detection of Cryptosporidium parvum and Cryptosporidium hominis in human patients in Cairo, Egypt. Parasitol Res 2012; 110(1): 161-166.

6. Helmy YA, Von Samson- Himmelstjerna G, Nöckler K, Zessin KH. Frequencies and spatial distributions of Cryptosporidium in livestock animals and children in the Ismailia province of Egypt. Epidemiol Infect 2015; 143(6):1208-1218.

7. Ghallab MMI, Aziz IZA, Shoeib EY, El-Badry AA. Laboratory utility of coproscopy, copro immunoassays and copro nPCR assay targeting Hsp90 gene for detection of Cryptosporidium in children, Cairo, Egypt. J Parasit Dis 2016; 40:901-905.

8. El-Badry AA, Abdel Aziz IZ, Shoeib EY, Ghallab MMI. Cryptosporidium genotypes and associated risk factors in a cohort of Egyptian children. Comp Clin Pathol 2017; 26:1017-1021.

9. Xiao L, Ryan UM. Cryptosporidiosis: an update in molecular epidemiology. Curr Opin Infect Dis 2004; 17(5): 483-490.

10. Cama VA, Bern C, Roberts J, Cabrera L, Sterling CR, Ortega $\mathrm{Y}$, et al. Cryptosporidium species and subtypes and clinical 
manifestations in children, Peru. Emerg Infect Dis 2008; 14(10):1567-1574.

11. Xiao L, Fayer R. Molecular characterization of species and genotypes of Cryptosporidium and Giardia and assessment of zoonotic transmission. Int J Parasitol 2008; 38(11):1239-1255.

12. Sadek GS. Use of nested PCR-RFLP for genotyping of Cryptosporidium parasites isolated from calves and children suffering from diarrhea. PUJ 2014; 7:129-137.

13. Henriksen SA, Pohlenz JF. Staining of cryptosporidia by a modified Ziehl-Neelsen technique. Acta Vet Scand 1981; 22(3-4):594-596.

14. Chen XM, Keithly JS, Paya CV, LaRusso NF. Cryptosporidiosis. New Eng J Med 2002; 34:1723-1731.

15. Abbaszadegan MR, Velayati A, Tavasoil A, Dadkhah E. Rapid DNA extraction protocol from stool, suitable for molecular genetics diagnosis of colon cancer. Iran Biomed J 2007; 11:203-208.

16. QIAamp Fast DNA Stool Mini Handbook. For fast purification of genomic DNA from stool samples. Catalog no. 51604. QIAGEN Sample and Assay Technologies. 2014.

17. Xiao L, Morgan UM, Limor J, Escalante A, Arrowood M, Shulaw W, et al. Genetic diversity within Cryptosporidium parvum and related Cryptosporidium species. Appl Environ Microbiol 1999; 65(8):3386-3391.

18. Xiao L, Lal AA, Jiang J. Detection, and differentiation of Cryptosporidium oocysts in water by PCR-RFLP. In Methods in Molecular Biology, Public Health Microbiology: Methods and Protocols, J.F.T. Spencer, A.L. Ragout de Spencer (eds). Humana Press Inc., Totowa, NJ, USA. 2004; pp. 163-176.

19. Liu L, Johnson HL, Cousens S, Perin J, Scott S, Lawn JE, et al. Global, regional, and national causes of child mortality: an updated systematic analysis for 2010 with time trends since 2000. Lancet 2012; 379(9832):2151-2161.

20. Striepen B. Parasitic infections: Time to tackle cryptosporidiosis. Nature 2013; 503(7475):189-191.

21. Kotloff KL, Nataro JP, Blackwelder WC, Nasrin D, Farag $\mathrm{TH}$, Panchalingam S, et al. Burden and aetiology of diarrhoeal disease in infants and young children in developing countries (the Global Enteric Multicenter Study, GEMS): a prospective, case-control study. Lancet 2013; 382(9888):209-222.

22. Adler S, Widerström M, Lindh J, Lilja M. Symptoms and risk factors of Cryptosporidium hominis infection in children: data from a large waterborne outbreak in Sweden. Parasitol Res 2017; 116(10):2613-2618.

23. Omoruyi BE, Nwodo UU, Udem CS, Okonkwo FO. Comparative diagnostic techniques for Cryptosporidium infection. Molecules 2014; 19(2):2674-2683.

24. Choudhry N, Petry F, van Rooijen N, McDonald V. A protective role for interleukin 18 in interferon $\gamma$-mediated innate immunity to Cryptosporidium parvum that is independent of natural killer cells. J Infect Dis 2012; 206(1):117-124.

25. Sanad MM, Al-Malki JS. Cryptosporidiosis among immunocompromised patients in Saudi Arabia. J Egypt Soc Parasitol 2007; 37:765-774.
26. Al-Qobati SA, Al-Maktari MT, Al-Zoa AM, Derhim M. Intestinal parasitosis among Yemeni patients with cancer, Sana'a, Yemen. J Egypt Soc Parasitol 2012; 42:727-734.

27. Quiroz ES, Bern C, MacArthur JR, Xiao L, Fletcher M, Arrowood MJ, et al. An outbreak of cryptosporidiosis linked to a food handler. J Infect Dis 2000; 181(2):695700 .

28. Lassen B, Ståhl M, Enemark HL. Cryptosporidiosis - an occupational risk and a disregarded disease in Estonia. Acta Vet Scand 2014; 56(1):36

29. Mac Kenzie WR, Hoxie NJ, Proctor ME, Gradus MS, Blair KA, Peterson DE, et al. A massive outbreak in Milwaukee of Cryptosporidium infection transmitted through the public water supply. N Engl J Med 1994; 331(3):161-167.

30. Widerström M, Schönning C, Lilja M, Lebbad M, Ljung T, Allestam G, et al. Large outbreak of Cryptosporidium hominis infection transmitted through the public water supply, Sweden. Emerg Infect Dis 2014; 20(4):581-589.

31. Yoder JS, Beach MJ, Centers for Disease Control and Prevention (CDC). Cryptosporidiosis surveillance-United States, 2003-2005. MMWR Surveill Summ 2007; 56(7):1-10.

32. El-Badry AA, Al-Antably ASA, Hassan MA, Hanafy NA, Abu-Sarea EY. Molecular seasonal, age and gender distributions of Cryptosporidium in diarrhoeic Egyptians: distinct endemicity. Eur J Clin Microbiol Infect Dis 2015; 34(12):2447-2453.

33. Hsu B, Huang C, Hsu C. Analysis for Giardia cysts and Cryptosporidium oocysts in water samples from small water systems in Taiwan. Parasitol Res 2001; 87:163168.

34. Al Hussainy NH. Prevalence of Cryptosporidium spp. infection in Saudi children. PUJ 2013; 6(2):149-156.

35. Amin OM. The epidemiology of Cryptosporidium parvum infections in the United States. PUJ 2008; 1(1):15-22.

36. Abdel Gawad SS, Ismail MAM, Imam NFA, Eassa AHA, AbuSarea EY. Detection of Cryptosporidium spp. in diarrheic immunocompetent patients in Beni-Suef, Egypt: Insight into epidemiology and diagnosis. Korean J Parasitol 2018; 56(2):113-119.

37. Helmy YA, Krücken J, Nöckler $K$, von SamsonHimmelstjerna G, Zessin KH. Molecular epidemiology of Cryptosporidium in livestock animals and humans in the Ismailia province of Egypt. Vet Parasitol 2013; 193(13):15-24.

38. Soliman RH, Othman AA. Evaluation of DNA melting curve Analysis real-time PCR for detection and differentiation of Cryptosporidium species. PUJ 2009; 2(1):47-54.

39. Sulaiman IM, Hira PR, Zhou L, Al-Ali FM, Al-Shelahi FA, Shweiki HM, et al. Unique endemicity of cryptosporidiosis in children in Kuwait. J Clin Microbiol 2005; 43(6):28052809.

40. Rafiei A, Rashno Z, Samarbafzadeh A, Khademvatan S. Molecular characterization of Cryptosporidium spp. isolated from immunocompromised patients and children. Jundishapur J Microbiol 2014; 7(4):e9183.

41. Dey A, Ghoshal U, Agarwal V, Ghoshal UC. Genotyping of Cryptosporidium species and their clinical manifestations in patients with renal transplantation and human 
immunodeficiency virus infection. J Pathog 2016; 2016: 2623602.

42. Elwin K, Hadfield SJ, Robinson G, Crouch ND, Chalmers RM. Cryptosporidium viatorum n. sp. (Apicomplexa: Cryptosporidiidae) among travelers returning to Great Britain from the Indian subcontinent, 2007-2011. Int J Parasitol 2012; 42(7):675-682.

43. Rossi P, Rivasi F, Codeluppi M, Catania A, Tamburrini A, Righi E, et al. Gastric involvement in AIDS associated cryptosporidiosis. Gut 1998; 43(4):476-477.

44. Ramsay DB, Long SE, Ali MA, Entwisle C, Orenstein JM, Rossi C, et al. Isolated gastric cryptosporidiosis in an immunocompetent patient. Dig Dis Sci 2007; 52(5):13641366.

45. Rani H, Gupta V, Gulati N, Chander J. Cryptosporidial oocysts in gastric aspirate of an infant. Indian J Med Microbiol 2009; 27(2):172-174.
46. DuPont HL, Chappell CL, Sterling CR, Okhuysen PC, Rose JB, Jakubowski W. The infectivity of Cryptosporidium parvum in healthy volunteers. N Engl J Med 1995; 332(13): 855-859.

47. Rehn M, Wallensten A, Widerström M, Lilja M, Grunewald $\mathrm{M}$, Stenmark S, et al. Post-infection symptoms following two large waterborne outbreaks of Cryptosporidium hominis in Northern Sweden, 2010-2011. BMC Public Health 2015; 15:529.

48. Hunter PR, Hughes S, Woodhouse S, Raj N, Syed Q Chalmers RM, et al. Sequelae of human cryptosporidiosis in immunocompetent patients. Clin Infect Dis 2004; 39(4):504-510.

49. Insulander M, Silverlås C, Lebbad M, Karlsson L, Mattsson JG, Svenungsson B. Molecular epidemiology and clinical manifestations of human cryptosporidiosis in Sweden. Epidemiol Infect 2013; 141(5):1009-1020. 